\title{
Variabilidade espacial da erosividade das chuvas no Brasil
}

\author{
Ana Luíza Ferreira Trindade(1), Paulo Tarso Sanches de Oliveira(2), \\ Jamil Alexandre Ayach Anache ${ }^{(1)}$ e Edson Wendland ${ }^{(1)}$
}

\begin{abstract}
(1)Universidade de São Paulo, Departamento de Hidráulica e Saneamento, Caixa Postal 359, CEP 13560-590 São Carlos, SP, Brasil. E-mail: ana.Iftrindade@gmail.com, anache.jamil@gmail.com, ew@sc.usp.br ${ }^{(2)}$ Universidade Federal de Mato Grosso do Sul, Caixa Postal 549, CEP 79070-900 Campo Grande, MS, Brasil. E-mail: paulotarsoms@gmail.com
\end{abstract}

\begin{abstract}
Resumo - O objetivo deste trabalho foi elaborar um novo mapa de erosividade da chuva para o Brasil, utilizando séries pluviométricas superiores a 20 anos, e analisar a distribuição espacial dos valores de erosividade. Dados de chuvas de 1.521 estações foram aplicados a 75 equações de regressão que relacionam a precipitação média anual $(\mathrm{P})$ e o coeficiente de chuvas $\left(\mathrm{R}_{\mathrm{c}}\right)$ com o índice de erosividade $\left(\mathrm{EI}_{30}\right)$. Os valores de erosividade para os locais não amostrados foram obtidos por interpolação, com uso do método de krigagem ordinária. Os índices mais elevados foram observados entre novembro e fevereiro. Os resultados consistem de interpolações que apresentam desempenho satisfatório para a estimativa da distribuição espacial da erosividade média anual das chuvas e do $\mathrm{EI}_{30}$, ao longo dos meses do ano. Assim, estes mapas podem auxiliar no planejamento da conservação do solo e da água, a partir do delineamento das regiões e das épocas do ano mais críticas quanto à erosividade da chuva.
\end{abstract}

Termos para indexação: conservação do solo e da água, $\mathrm{EI}_{30}$, krigagem, USLE.

\section{Spatial variability of rainfall erosivity in Brazil}

\begin{abstract}
The objective of this work was to develop a new rainfall erosivity map for Brazil, using rainfall time series longer than 20 years, and to analyze the spatial distribution of erosivity values. Rainfall data from 1,521 rain gauges were applied to 75 regression equations that relate the mean annual precipitation $(\mathrm{P})$ and the rainfall coefficient $\left(\mathrm{R}_{\mathrm{c}}\right)$ with the rainfall erosivity index $\left(\mathrm{EI}_{30}\right)$. Rainfall erosivity values for ungauged regions were obtained by interpolation using the ordinary kriging method. The highest indices were observed between November and February. The results are interpolations that show satisfactory performance for estimating the spatial distribution of the mean annual rainfall erosivity and the $\mathrm{EI}_{30}$ during the months of the year. Therefore, those maps can help on soil and water conservation planning, considering the design of those areas and periods of the year that are considered more critical for rainfall erosivity in Brazil.
\end{abstract}

Index terms: soil and water conservation, $\mathrm{EI}_{30}$, kriging, USLE.

\section{Introdução}

A produção agropecuária no ano de 2015 correspondeu a $23 \%$ do produto interno bruto (PIB) gerado no Brasil (Brasil, 2016). No entanto, a erosão hídrica ameaça essa produção, acarretando problemas como a perda de água, solo, nutrientes e matéria orgânica, degradação do solo, diminuição da produtividade e poluição dos corpos d'água (Pimentel, 2006). Assim, a erosão do solo tem sido considerada uma grande ameaça aos recursos ambientais e à produção de alimentos (Oliveira et al., 2015).

Estima-se que a degradação do solo custe US\$ 10,6 trilhões anualmente, em todo o mundo, o que equivale a 17\% do PIB global (Stewart, 2015). Nos Estados Unidos, o custo econômico da erosão do solo foi estimado entre US\$ 30 bilhões (Uri \& Lewis, 1998) e US\$ 44 bilhões (Pimentel et al., 1995), enquanto na União Europeia foi de US\$ 45,5 bilhões (Montanarella, 2007). No Brasil, Telles et al. (2011) relataram valores de US\$ 242 milhões e US\$ 212 milhões para os Estados do Paraná e São Paulo, respectivamente. Assim, estudos sobre erosão hídrica são fundamentais para a sustentabilidade da produção agropecuária.

A erosão hídrica é causada pelo impacto das gotas de chuvas que promovem a desagregação de partículas do solo que, posteriormente, são transportadas pelo escoamento superficial. Conhecer o potencial erosivo das chuvas é crucial para estimar a perda de solo (Shamshad et al., 2008; Carvalho et al., 2014). Uma das formas de estimar o potencial erosivo das chuvas é o uso de índices de erosividade como o $\mathrm{EI}_{30}$ proposto na USLE (universal soil loss equation). O potencial erosivo 
está relacionado às características físicas das chuvas, como quantidade, intensidade, intensidade máxima em $30 \mathrm{~min}$, diâmetro das gotas, velocidade terminal e energia cinética (Wischmeier \& Smith, 1978). No entanto, estes parâmetros são escassos e de difícil obtenção, pois necessitam de dados pluviográficos de alta resolução e de medição contínua por um longo período de tempo.

No Brasil, faltam informações espaço-temporais para o cálculo do índice de erosividade, o que dificulta sua obtenção em diversas localidades. Portanto, a utilização de modelos capazes de estimar os valores de erosividade, a partir de dados mais acessíveis, como média mensal ou anual de precipitação, constitui uma importante ferramenta para a tomada de decisão de práticas de conservação do solo e da água (Lee \& Heo, 2011; Oliveira et al., 2013). Além disso, o índice também pode servir como indicador de mudanças climáticas e seus potenciais efeitos sobre os processos erosivos (Biasutti \& Seager, 2015; Hoomehr et al., 2016).

No Brasil, diversos autores buscaram relacionar o índice de erosividade com dados pluviométricos, por meio de equações de regressão, como uma maneira de estimar os valores de erosividade para locais onde não há cobertura de dados pluviográficos (Mello et al., 2007; Oliveira et al., 2012; Eltz et al., 2013). Um levantamento detalhado de trabalhos sobre erosividade no país foi realizado por Oliveira et al. (2013), com o intuito de analisar a qualidade dos resultados obtidos e sua representatividade, o que contribuiu para a melhor compreensão do potencial erosivo das chuvas no Brasil.

Embora a utilização de equações de regressão permita estimar valores para o índice de erosividade em diversos locais, ainda haverá regiões onde medições simples de chuvas não são realizadas e, portanto, não haverá séries de dados pluviométricos para aplicar às equações até então desenvolvidas. Os valores de erosividade para estas áreas podem ser estimados por meio da aplicação de métodos de interpolação baseados nos valores amostrados (Montebeller et al., 2007). Entre as várias técnicas de interpolação, podem-se citar: o método do inverso do quadrado da distância; o método das isoietas; e métodos geoestatísticos. Assim, ferramentas como sistemas de informações geográficas (SIG), sensoriamento remoto, geoestatística, análises de regressão e técnicas de análise multivariada têm sido utilizadas no mapeamento da erosividade média anual, em diversos locais no mundo (Diodato et al., 2013; Mello et al., 2013; Panagos et al., 2015; Schmidt et al., 2016), gerando discussões sobre qual ou quais métodos são mais adequados para tais estimativas.

Os métodos de interpolação podem ser aplicados à elaboração de mapas de erosividade, para fornecer informações importantes sobre o potencial de erosão da chuva em uma região e auxiliar a implementação de medidas necessárias para a redução da erosão do solo. Silva (2004) elaborou um mapa de erosividade do Brasil, a partir de dados pluviométricos de 1.600 estações, por meio de séries históricas de precipitação de no mínimo 10 anos. No entanto, o autor utilizou oito equações de regressão representativas para o cálculo da erosividade regional, a partir das médias mensais e anuais de chuvas. Mello et al. (2013) usaram dados pluviométricos de 773 estações, com no mínimo 15 anos de dados, para estimar a erosividade da chuva no Brasil. Na obtenção do mapa de erosividade anual média para o país, foram integradas técnicas de análise multivariada e geoestatística para a modelagem dos dados, em que se considerou um número muito maior de equações de erosividade (54) em comparação ao mapa de Silva (2004). Entretanto, avanços podem ser feitos, aumentando-se o número de equações de erosividade e o uso de maiores séries de dados históricos de chuva, para estimar o fator R no Brasil, pois, a falta de dados em regiões do país como o Centro-Oeste e o Norte prejudica os resultados finais das análises. Renard et al. (1997) recomendam que sejam usados, no mínimo, 20 anos de dados de chuva para tais estimativas. Além disso, outras abordagens do processo de modelagem podem ser consideradas, como a maneira de definir qual equação de erosividade se deve utilizar para cada série histórica de dados pluviométricos.

O objetivo deste trabalho foi elaborar um novo mapa de erosividade da chuva para o Brasil, por meio do uso de séries pluviométricas superiores a 20 anos, e analisar a distribuição espacial dos valores de erosividade.

\section{Material e Métodos}

O estudo foi desenvolvido para todo o território brasileiro, que compreende aproximadamente 850 milhões de hectares. Primeiramente, foram levantados os dados de precipitação. Considerando-se as 8.587 estações pluviométricas fornecidas pela Agência 
Nacional das Águas, por meio do HidroWeb (2015), somente 1.521 estações apresentaram série histórica igual ou superior a 20 anos, conforme recomendado por Renard et al. (1997), o que representa aproximadamente $20 \%$ do total de estações disponibilizadas. Assim, foram identificados os seguintes parâmetros: as estações pluviométricas que cumprem os requisitos do estudo; o total de anos que elas representam em sua série histórica; a média de precipitação anual e mensal de cada estação; e a média de precipitação de cada mês. Posteriormente, as estações selecionadas foram inseridas em um sistema de informações geográficas (SIG), uma vez que a cada uma foi atribuída a sua coordenada geográfica.

Além disso, realizou-se uma análise das estações por bacias hidrográficas brasileiras (Figura 1), pois modelos como a USLE, quando utilizados neste tipo de escala, integram detalhes topográficos e simplificam a detalhada malha hidrográfica, o que possibilita esta adaptação de escala de forma mais consistente (Kirby et al., 1996). Para o parâmetro erosividade, a abordagem em escala de bacia hidrográfica é justificada pelo fato de este tipo de unidade territorial integrar os diversos fatores hidrológicos que envolvem os processos de erosão hídrica.
Posteriormente, aplicaram-se 75 equações de regressão para a estimativa da erosividade da chuva, utilizando-se os dados destas estações conforme os procedimentos detalhados a seguir. Estas equações foram levantadas por Oliveira et al. (2013), que desenvolveram um estudo de revisão que reuniu todas as equações disponíveis nas bases de dados científicos mais acessadas atualmente, distribuídas em diferentes localidades. Estas equações foram utilizadas para o cálculo da erosividade e, posteriormente, definiuse qual equação seria utilizada para cada estação pluviométrica (1.521 postos da ANA). As áreas de influência das equações de erosividade (devidamente georreferenciadas) foram determinadas a partir do método dos polígonos de Thiessen, gerados em ambiente SIG pelo programa ArcGIS 10.2 (Environmental Systems Research Institute, Redlands, CA, EUA). Cada polígono de Thiessen (gerado a partir do conjunto de pontos de localização das estações que geraram as equações) contém um único ponto. Assim, qualquer local dentro do polígono está mais próximo do seu ponto central correspondente do que de qualquer outro ponto utilizado para a construção do conjunto de polígonos. Os parâmetros necessários para o cálculo da erosividade de uma determinada equação

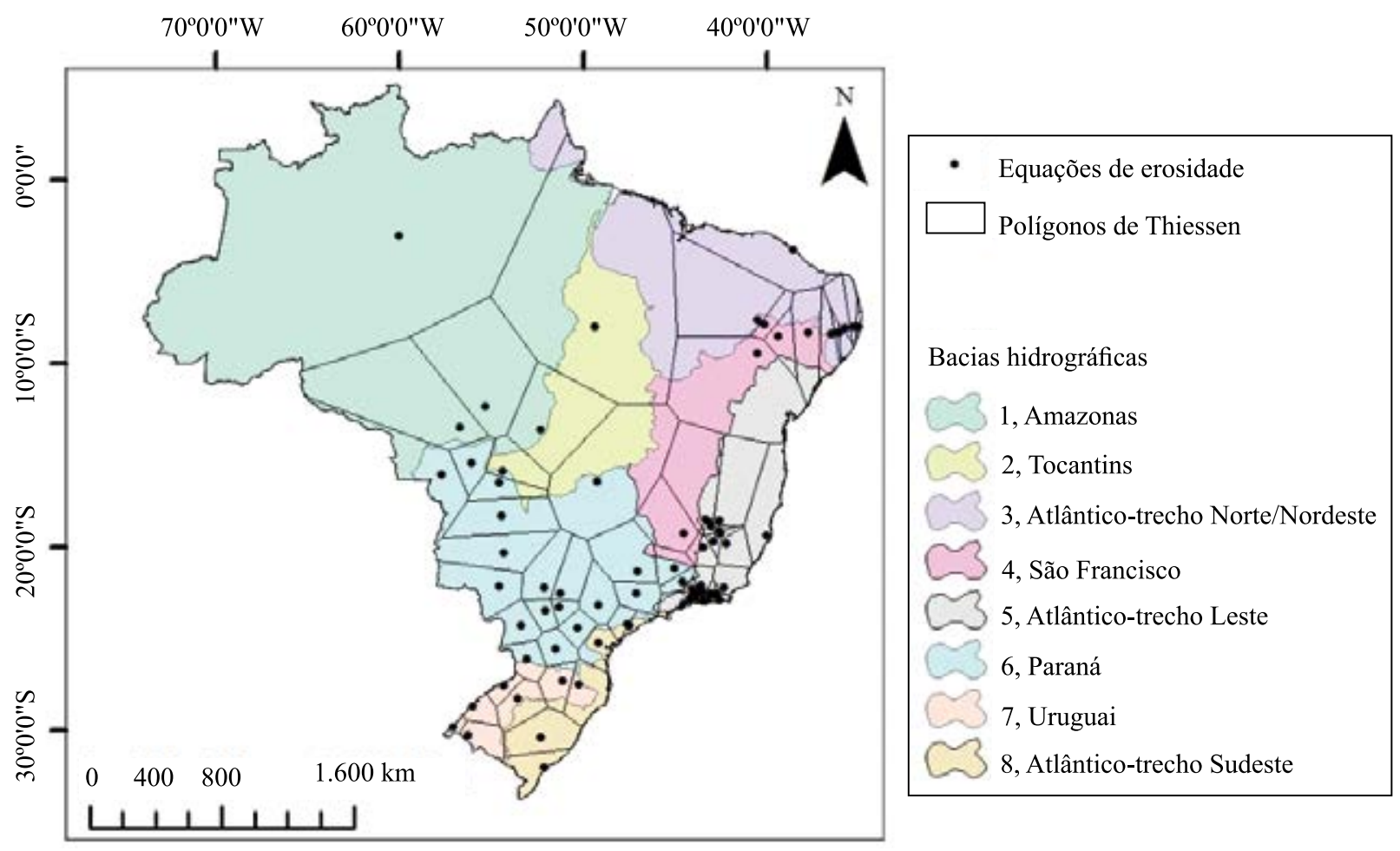

Figura 1. Grandes bacias hidrográficas do Brasil e suas áreas de influência das equações de erosividade. 
foram definidos a partir das estações pluviométricas que estão localizadas dentro da área de influência daquela equação.

As médias mensais e anuais de 1.521 estações pluviométricas do banco de dados foram utilizadas para posterior aplicação às equações de regressão no cálculo da erosividade. Para o cálculo da erosividade, as 75 equações de regressão usadas levaram em conta o coeficiente de chuva (quadrado da precipitação média mensal dividido pela precipitação média anual) ou somente a precipitação média mensal (Oliveira et al., 2013). Em alguns estudos apresentados por Oliveira et al. (2013), foram desenvolvidas pelo menos duas equações, por meio de diferentes técnicas de regressão simples, para o cálculo do índice de erosividade. Para cada equação descrita, foi também apresentado o coeficiente de determinação encontrado $\left(R^{2}\right)$ pelos respectivos autores, entre os valores anuais de erosividade e os parâmetros de entrada da equação (precipitação média anual ou coeficiente de chuvas). Nestes casos, optou-se inicialmente pela equação que apresentou o maior valor de $\mathrm{R}^{2}$ para o cálculo do $\mathrm{EI}_{30}$. Após a obtenção dos valores de $\mathrm{EI}_{30}$ para cada mês do ano, o índice de erosividade anual médio (fator $\mathrm{R} d a$ USLE) foi calculado a partir do somatório dos valores de erosividade mensais.

Para a elaboração dos mapas de erosividade do Brasil, atribuíram-se os valores de $\mathrm{EI}_{30}$ (mensal) e o fator $\mathrm{R}$ ( $\mathrm{EI}_{30}$ anual médio) às coordenadas geográficas das estações pluviométricas selecionadas. Em seguida, os dados de erosividade foram interpolados por meio de krigagem ordinária, utilizando-se os dados sem nenhum tipo de transformação, com o objetivo de estimar os valores de erosividade para os locais não amostrados. Para isso, utilizou-se o pacote geoestatístico do ArcGIS 10.2 que, além das interpolações, ajustou os semivariogramas para um modelo do tipo estável (stable). A erosividade anual do Brasil foi obtida a partir da interpolação dos valores do fator R, enquanto as interpolações das erosividades mensais foram obtidas a partir dos valores de $\mathrm{EI}_{30}$ referentes a cada mês do ano. Os mapas foram gerados posteriormente também pelo ArcGIS 10.2, e as escalas de cores foram padronizadas para possibilitar a comparação entre os mapas obtidos. Além disso, o mapa do fator R foi classificado de acordo com Foster et al. (1981).
A validação do mapa de erosividade anual gerado foi realizada por meio de validação cruzada (crossvalidation). $\mathrm{O}$ método consiste da remoção de um valor componente da base de dados e da realização da estimativa para as regiões não amostradas, como é feito usualmente. Em seguida, faz-se uma comparação entre o valor real e o estimado para aquele ponto removido. O procedimento é repetido para todos os pontos da base de dados. Para a validação, os valores estimados de erosividade foram comparados aos valores amostrados (observados), por meio de métricas estatísticas, como: o coeficiente de determinação $\left(\mathrm{R}^{2}\right)$, o coeficiente de eficiência de Nash-Sutcliffe (NSE) e o erro médio absoluto (MAE).

\section{Resultados e Discussão}

As estações pluviométricas concentram-se no litoral, especialmente das regiões Sul e Sudeste, enquanto as regiões Norte e Centro-Oeste têm uma cobertura precária de monitoramento das chuvas (Figura $2 \mathrm{e}$ Tabela 1). Em particular, a Bacia Amazônica apresenta a maior área territorial, no entanto, apresentou a menor

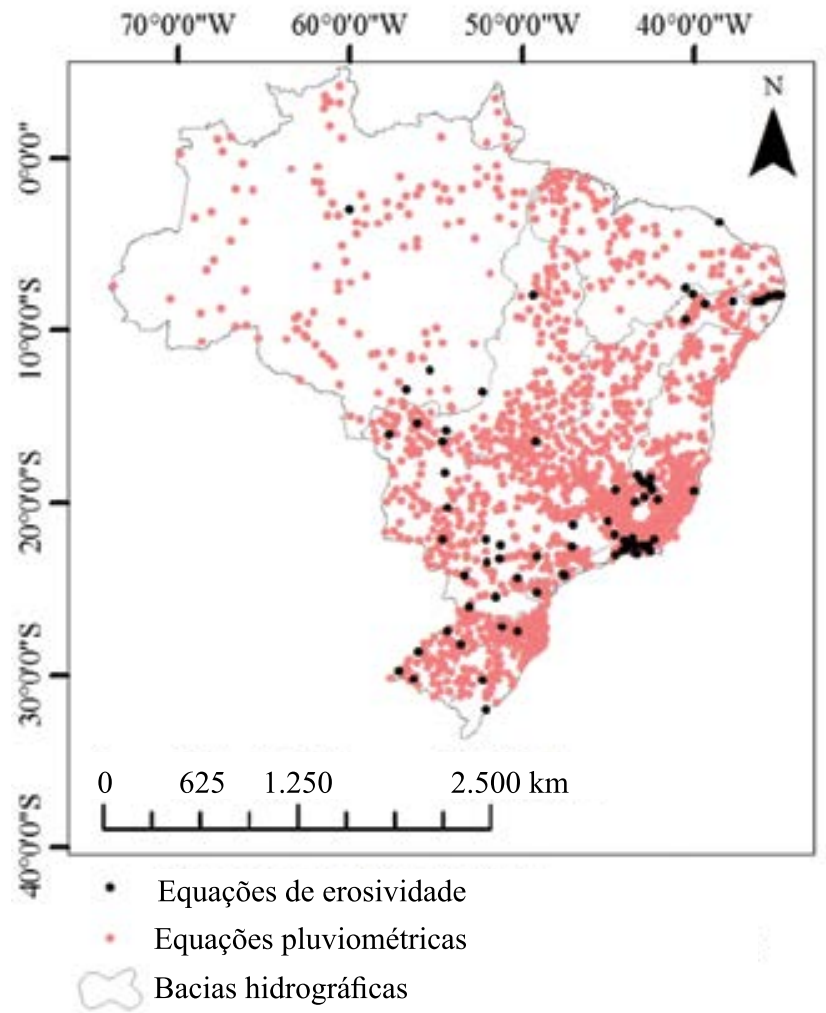

Figura 2. Estações pluviométricas selecionadas para o cálculo do índice de erosividade.

Pesq. agropec. bras., Brasília, v.51, n.12, p.1918-1928, dez. 2016 DOI: $10.1590 / \mathrm{S} 0100-204 X 2016001200002$ 
série histórica de dados e de densidade de estações, em relação à área. Esses resultados corroboram os apresentados por Melo et al. (2015) e reforçam a necessidade de expansão da rede de monitoramento hidrológico brasileiro. As Tabelas 2, 3 e 4 apresentam as precipitações médias mensais e anuais e o coeficiente de chuvas médio obtido em cada bacia hidrográfica. Ao analisar as precipitações médias de cada mês (Tabela 2), nota-se, em geral, maiores precipitações, entre os meses de janeiro e março, e um período de estiagem

Tabela 1. Extensão média das séries históricas de dados e densidade de estações por bacia.

\begin{tabular}{|c|c|c|c|c|}
\hline Bacia hidrográfica & $\begin{array}{l}\text { Série histórica média } \\
\text { (anos) }\end{array}$ & $\begin{array}{c}\text { Área da bacia } \\
\left(10^{6} \mathrm{~km}^{2}\right)\end{array}$ & Estações selecionadas & $\begin{array}{c}\text { Estações por unidade de área } \\
\text { (unid. } 10^{-6} \mathrm{~km}^{2} \text { ) }\end{array}$ \\
\hline 1, Amazonas & 24 & 3,870 & 123 & 32 \\
\hline 2, Tocantins-Araguaia & 31 & 0,919 & 161 & 175 \\
\hline 3, Atlântico - Trecho N/NE & 32 & 0,894 & 130 & 145 \\
\hline 4, São Francisco & 39 & 0,639 & 249 & 390 \\
\hline 5, Atlântico - Trecho S & 39 & 0,603 & 333 & 552 \\
\hline 6, Paraná & 33 & 1,243 & 372 & 299 \\
\hline 7, Uruguai & 35 & 0,175 & 110 & 630 \\
\hline 8, Atlântico - Trecho SE & 40 & 0,188 & 43 & 229 \\
\hline
\end{tabular}

Tabela 2. Médias mensais de precipitação por bacia hidrográfica.

\begin{tabular}{|c|c|c|c|c|c|c|c|c|c|c|c|c|c|}
\hline \multirow[t]{2}{*}{$\overline{\text { Bacia }}$} & \multirow{2}{*}{$\begin{array}{c}\text { Precipitação média } \\
\text { mensal } \pm \text { dp (mm) }\end{array}$} & \multicolumn{12}{|c|}{ Precipitação mensal (mm) } \\
\hline & & Jan. & Fev. & Mar. & Abr. & Maio & Jun. & Jul. & Ago. & Set. & Out. & Nov. & Dez. \\
\hline$\overline{1}$ & $178,61 \pm 31,54$ & 263,67 & 261,63 & 281,11 & 240,66 & 186,34 & 120,69 & 91,65 & 79,93 & 96,50 & 135,42 & 170,00 & 215,03 \\
\hline 2 & $130,92 \pm 6,49$ & 279,05 & 234,34 & 232,67 & 119,47 & 35,43 & 7,89 & 4,22 & 9,12 & 44,64 & 127,64 & 205,64 & 270,94 \\
\hline 3 & $126,59 \pm 2,53$ & 190,09 & 209,41 & 274,52 & 243,08 & 164,97 & 95,95 & 75,53 & 45,21 & 32,46 & 36,07 & 50,99 & 100,85 \\
\hline 4 & $92,26 \pm 24,44$ & 203,57 & 138,70 & 142,06 & 62,00 & 27,73 & 13,44 & 12,11 & 10,18 & 29,35 & 79,06 & 166,19 & 222,77 \\
\hline 5 & $104,81 \pm 29,51$ & 175,28 & 118,49 & 139,98 & 94,45 & 64,41 & 47,93 & 48,78 & 40,73 & 58,86 & 99,83 & 167,97 & 200,96 \\
\hline 6 & $119,88 \pm 15,11$ & 235,09 & 189,14 & 170,01 & 91,39 & 66,05 & 39,16 & 27,57 & 31,48 & 72,58 & 126,86 & 166,78 & 222,45 \\
\hline 7 & $144,26 \pm 16,18$ & 154,36 & 153,46 & 131,82 & 145,76 & 136,43 & 132,60 & 134,44 & 120,85 & 154,30 & 182,97 & 144,22 & 139,93 \\
\hline 8 & $131,94 \pm 19,93$ & 182,13 & 175,82 & 146,28 & 109,15 & 103,19 & 103,77 & 113,50 & 102,48 & 134,31 & 143,87 & 122,08 & 146,70 \\
\hline Média & - & 210,41 & 185,12 & 189,81 & 138,25 & 98,07 & 70,18 & 63,47 & 55,00 & 77,87 & 116,47 & 149,23 & 189,95 \\
\hline
\end{tabular}

Tabela 3. Médias anuais de precipitação e coeficiente médio de chuva por bacia hidrográfica.

\begin{tabular}{|c|c|c|c|c|c|c|c|c|c|c|c|c|c|}
\hline \multirow[t]{2}{*}{ Bacia } & \multirow{2}{*}{$\begin{array}{l}\text { Precipitação média } \\
\text { anual }(\mathrm{mm})\end{array}$} & \multicolumn{12}{|c|}{ Coeficiente de chuva $\mathrm{R}_{\mathrm{c}}(\mathrm{mm})$} \\
\hline & & Jan. & Fev. & Mar. & Abr. & Maio & Jun. & Jul. & Ago. & Set. & Out. & Nov. & Dez. \\
\hline$\overline{1}$ & $2.143,29$ & 35,72 & 34,43 & 39,24 & 28,67 & 20,01 & 11,49 & 7,53 & 4,81 & 4,87 & 9,70 & 15,62 & 24,86 \\
\hline 2 & $1.571,06$ & 50,17 & 35,08 & 34,71 & 9,94 & 1,03 & 0,06 & 0,02 & 0,07 & 1,36 & 10,51 & 27,36 & 47,50 \\
\hline 3 & $1.519,12$ & 25,30 & 30,04 & 52,07 & 40,12 & 19,46 & 8,63 & 6,42 & 2,22 & 0,98 & 1,16 & 2,65 & 8,16 \\
\hline 4 & $1.107,16$ & 38,36 & 17,73 & 19,23 & 4,18 & 1,34 & 0,76 & 0,60 & 0,26 & 0,91 & 6,01 & 26,08 & 46,43 \\
\hline 5 & $1.257,68$ & 26,46 & 11,80 & 15,92 & 7,76 & 4,75 & 3,19 & 3,04 & 1,88 & 3,01 & 8,22 & 24,04 & 34,79 \\
\hline 6 & $1.438,58$ & 39,57 & 25,38 & 20,72 & 6,06 & 3,69 & 1,71 & 0,91 & 0,97 & 4,05 & 11,39 & 19,85 & 35,49 \\
\hline 7 & $1.731,15$ & 13,88 & 13,76 & 10,19 & 12,84 & 10,87 & 10,24 & 10,59 & 8,67 & 13,86 & 19,47 & 12,10 & 11,44 \\
\hline 8 & $1.583,28$ & 21,94 & 20,26 & 14,09 & 7,83 & 6,88 & 7,29 & 8,77 & 7,09 & 11,59 & 13,18 & 9,48 & 13,91 \\
\hline
\end{tabular}

Tabela 4. Índices de erosividade anual e mensal das bacias hidrográficas brasileiras.

\begin{tabular}{|c|c|c|c|c|c|c|c|c|c|c|c|c|c|}
\hline \multirow[t]{2}{*}{ Bacia } & \multirow[t]{2}{*}{ Fator R } & \multicolumn{12}{|c|}{ Erosividade média mensal EI ${ }_{30}\left(\mathrm{MJ} \mathrm{mm} \mathrm{ha}^{-1} \mathrm{~h}^{-1}\right.$ por mês $)$} \\
\hline & & Jan. & Fev. & Mar. & Abr. & Maio & Jun. & Jul. & Ago. & Set. & Out. & Nov. & Dez. \\
\hline 1 & $12.488,81$ & $1.513,63$ & $1.520,59$ & $1.710,65$ & $1.443,65$ & $1.098,23$ & 739,32 & 583,83 & 499,40 & 552,81 & 742,91 & 915,09 & $1.168,72$ \\
\hline 2 & $10.274,21$ & $1.869,59$ & $1.452,21$ & $1.448,86$ & 637,62 & 274,69 & 213,56 & 208,20 & 217,02 & 304,67 & 659,62 & $1.197,56$ & $1.790,51$ \\
\hline 3 & $7.677,58$ & 921,57 & $1.073,28$ & $1.590,58$ & $1.306,65$ & 781,20 & 406,62 & 325,30 & 218,70 & 186,47 & 199,71 & 248,87 & 418,62 \\
\hline 4 & $7.446,74$ & $1.551,66$ & 866,27 & 898,19 & 247,44 & 96,30 & 66,00 & 62,51 & 56,45 & 109,03 & 395,97 & $1.238,19$ & $1.858,74$ \\
\hline 5 & $7.873,74$ & $1.229,99$ & 723,84 & 877,17 & 520,56 & 325,79 & 229,88 & 249,53 & 201,85 & 308,63 & 604,45 & $1.139,07$ & $1.462,98$ \\
\hline 6 & $7.924,72$ & $1.497,66$ & $1.080,59$ & 916,44 & 409,67 & 312,05 & 192,23 & 143,55 & 152,70 & 324,08 & 630,47 & 891,61 & $1.373,67$ \\
\hline 7 & $8.703,66$ & 775,05 & 782,76 & 652,17 & 847,08 & 680,82 & 629,58 & 617,78 & 511,72 & 773,54 & $1.055,94$ & 720,11 & 657,12 \\
\hline 8 & $6.528,70$ & 872,59 & 802,20 & 634,79 & 431,21 & 393,87 & 397,27 & 428,47 & 382,44 & 517,82 & 573,64 & 476,53 & 617,87 \\
\hline
\end{tabular}


entre julho e setembro. Destacam-se desta análise as bacias 7 (Uruguai) e 8 (Atlântico - trecho SE), que se encontram na região Sul do País, em que os índices de chuvas se mantêm praticamente constantes ao longo do ano, sem uma distinção clara entre as estações úmidas e secas.

Como esperado, os maiores valores anuais de precipitação foram registrados na Bacia Amazônica (bacia 1), que apresentou precipitação média mínima de aproximadamente $80 \mathrm{~mm}$ no mês de agosto, máxima de $281 \mathrm{~mm}$ em março e precipitação média anual de $2.143 \mathrm{~mm}$. As menores medições anuais de chuvas foram registradas na bacia 4 (Rio São Francisco), cuja área está, em parte, inserida na região do Semiárido Nordestino. Nesta bacia, a precipitação máxima mensal foi de $222 \mathrm{~mm}$, no mês de dezembro, mínima de $10 \mathrm{~mm}$ no mês de agosto e precipitação média anual de $1.107 \mathrm{~mm}$. No entanto, os menores valores de precipitação mensal foram encontrados na bacia 2 , do Rio Tocantins-Araguaia, que apresentou em julho média de 4,22 $\mathrm{mm}$. A mesma bacia também registrou o maior valor de precipitação mensal, no valor de 279 $\mathrm{mm}$ no mês de janeiro.

A distribuição de chuvas ao longo do ano é o principal fator que influencia os valores dos coeficientes de chuvas, conforme a Tabela 3, também observado por Mello et al. (2013) e Panagos et al. (2015). A bacia 2, por exemplo, apresenta os maiores e os menores valores dos coeficientes de chuvas entre todas as bacias, assim como também apresenta as maiores e menores medições de chuvas. É importante ressaltar que o potencial erosivo das chuvas não depende somente de sua quantidade, e que sua intensidade é o principal parâmetro para determinar o valor da erosividade. Por isso, é relevante destacar que a concentração de chuvas em determinados períodos do ano pode interferir significativamente nos valores calculados da erosividade.

$\mathrm{Na}$ Tabela 4, estão apresentados os valores de erosividade da chuva para cada bacia hidrográfica. Nota-se tendência de maiores valores de erosividade na Bacia do Rio Amazonas e menores na Bacia do Atlântico - trecho SE. Os resultados não interpolados da erosividade anual, estimados para todo o Brasil, variaram de 1.409 a $22.816 \mathrm{MJ} \mathrm{mm} \mathrm{ha}^{-1} \mathrm{~h}^{-1}$ por ano. Após a interpolação, o fator $\mathrm{R}$ variou entre $1.782 \mathrm{e}$ 16.583 MJ mm ha-1 $\mathrm{h}^{-1}$ por ano. Silva (2004), Mello et al. (2013) e Oliveira et al. (2013) verificaram valores de erosividade no país que variaram de 3.116 a 20.035 MJ mm ha-1 $\mathrm{h}^{-1}$ por ano, 2.216 a $23.187 \mathrm{MJ} \mathrm{mm} \mathrm{ha}^{-1} \mathrm{~h}^{-1}$ por ano e de 1.672 a $22.452 \mathrm{MJ} \mathrm{mm} \mathrm{ha}^{-1} \mathrm{~h}^{-1}$ por ano, respectivamente. Assim, os resultados corroboram parcialmente os trabalhos anteriores. A diferença das estimativas pode ser justificada pela utilização de uma rede mais densa de dados pluviométricos e equações de regressão para a obtenção da erosividade, que possibilitaram a utilização das equações com dados pluviométricos oriundos da mesma localidade, na maioria dos casos, o que reduziu a variação da interpolação proposta. No estudo de Mello et al. (2013), por exemplo, foram consideradas 54 equações de erosividade, contra as atuais 75 , que foram alimentadas pelos dados pluviométricos de 928 postos, contra os atuais 1.521, quantidades que são 39 e $64 \%$ maiores, respectivamente. Assim, as estimativas de erosividade no País tornam-se mais confiáveis.

No processo de krigagem ordinária, adotouse o modelo do tipo estável com alcance de $0,11^{\circ}$ (Figura 3) para o ajuste do semivariograma. O mapa de erosividade do Brasil foi elaborado a partir da superfície gerada por meio de método geoestatístico e também está apresentado em classes de erosividade, para facilitar a visualização dos resultados (Figura 4). Observa-se que $56 \%$ do País apresenta o fator $\mathrm{R}$ alto ou muito alto, contra $69 \%$ levantados por Silva (2004), além de a variação dos dados ter diminuído $13 \%$, em relação aos resultados de Silva (2004), e 29\% em relação aos números de Mello et al. (2013). Além disso, a distribuição das classes de erosividade foi mais heterogênea em relação aos referidos trabalhos, tendose detectado no mínimo $15 \%$ de superfície para cada classe de erosividade.

Logo, a espacialização da erosividade média anual permite identificar, principalmente, os locais que merecem atenção nas práticas conservacionistas do solo onde os índices pluviométricos são altos praticamente o ano todo. De acordo com os resultados obtidos, a região Norte apresenta os maiores índices de erosividade, que variam de 7.116 a 16.583 MJ mm ha ${ }^{-1} \mathrm{~h}^{-1}$ por ano. O Estado do Amapá, em especial, apresenta os maiores índices de erosividade média anual do País, com valor mínimo de 12.864 $\mathrm{MJ} \mathrm{mm} \mathrm{ha}{ }^{-1} \mathrm{~h}^{-1}$ por ano. No entanto, os menores índices de erosividade encontram-se na região Nordeste do País e variam entre 1.782 e $13.810 \mathrm{MJ} \mathrm{mm} \mathrm{ha}^{-1} \mathrm{~h}^{-1}$ por ano, o que concorda com estimativas anteriores de Silva (2004), Mello et al. (2013) e Oliveira et al. (2013).

Pesq. agropec. bras., Brasília, v.51, n.12, p.1918-1928, dez. 2016 DOI: 10.1590/S0100-204X2016001200002 
É possível observar que, nas regiões próximas à linha do Equador, os índices de erosividade e os de precipitação aumentam, conforme a longitude, na direção Oeste. Assim, é possível notar como a bacia 2, que abrange o Estado do Amapá e se estende desde a Ilha do Marajó até o litoral nordestino, pode apresentar os maiores e os menores valores de erosividade e de precipitação ao mesmo tempo. Em geral, o mapa de erosividade anual das chuvas apresentou os aspectos esperados, em que os valores de erosividade se relacionam aos índices pluviométricos observados nas regiões Norte e Nordeste do país, que apresentam os maiores e menores índices de erosividade, respectivamente.

O mapa de erosividade anual apresentou estimativas inesperadas da área que abrange o norte do Estado de Minas Gerais e o sul da Bahia. Trata-se de região classificada como semiárida, cujos valores de erosividade estão na faixa de 12.000 a 14.000 MJ mm ha ${ }^{-1} \mathrm{~h}^{-1}$ por ano, embora tenha apresentado baixos valores de precipitação média anual (Tabela 4). Tal incompatibilidade é atribuída à determinação das áreas de influência das equações de erosividade que utilizam o método de Thiessen. A área em questão corresponde ao polígono determinado ao redor da equação determinada por Silva et al. (2010) para o Município de Santo Antônio do Itambé, MG, cujo potencial erosivo das chuvas é de $15.280 \mathrm{MJ}$ $\mathrm{mm} \mathrm{ha} \mathrm{h}^{-1} \mathrm{~h}^{-1}$ por ano. Logo, os valores obtidos pela interpolação, nesta área de influência, aproximam-se do valor de erosividade da cidade de origem (Santo Antônio do Itambé, MG) e estão acima do esperado para a região norte de Minas Gerais e sul da Bahia. Estes resultados evidenciam a importância de estudos locais, para a obtenção dos índices de erosividade, e permitem gerar relações entre erosividade e precipitação que representem as particularidades de cada região, conforme realizado por Mazurana et al (2009), Almeida et al. (2012) e Eltz et al. (2013). Além disso, esta incompatibilidade deixa clara a limitação associada ao método de Thiessen para a delimitação das áreas de influência das equações.

A evolução da erosividade mensal (Figura 5) assemelha-se à distribuição da precipitação ao longo do ano, quando é possível distinguir claramente as estações chuvosas e secas (Tabela 2), e permite identificar as épocas do ano em que a exposição do solo ou a ocupação inadequada pode contribuir para

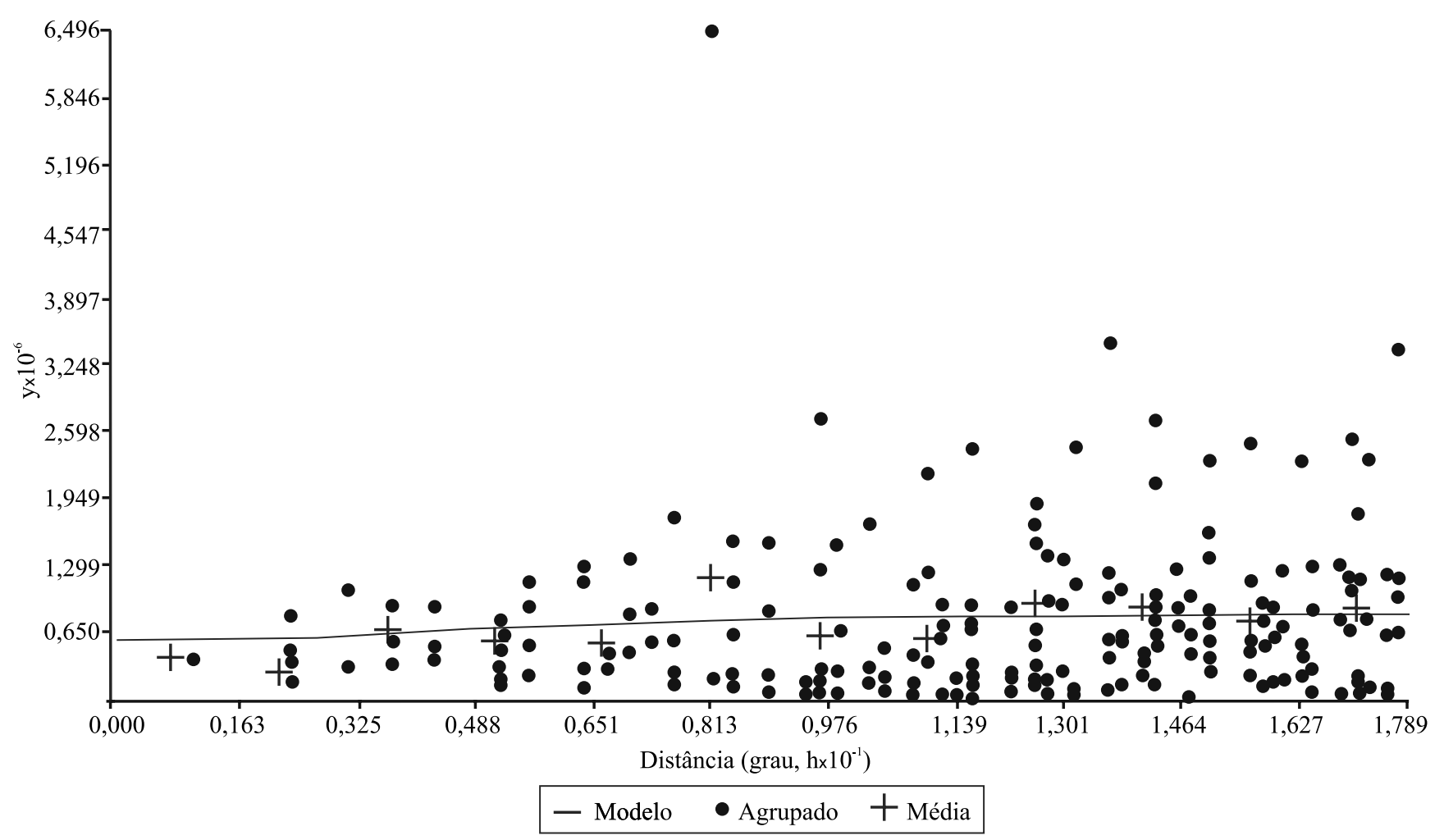

Figura 3. Semivariograma ajustado por meio de krigagem ordinária. 
aumento da erosão em determinados locais. Nos meses mais chuvosos (entre novembro e março), é possível observar altos índices de erosividade em quase todo o território brasileiro, com destaque para a região Norte do país, que apresenta os maiores índices de precipitação e erosividade praticamente o ano todo. Excetuam-se desta observação a porção da região Nordeste também conhecida como polígono da seca e boa parte da área da bacia 8 , localizada na região Sul do Brasil, como também observado por Oliveira et al. (2013). No caso do Nordeste, os índices pluviométricos não são expressivos durante o ano, mesmo na estação das chuvas e, por isso, os índices de erosividade sempre apresentaram valores inferiores aos das demais regiões do país. Na bacia 8 , localizada no litoral sul do País, embora a precipitação média anual seja uma das mais elevadas em relação às demais bacias hidrográficas, as precipitações mensais estão distribuídas de maneira uniforme no ano (Tabela 3). Este aspecto se refletiu nos valores dos coeficientes de chuvas (Tabela 4) $\mathrm{e}$, consequentemente, na estimativa dos índices de erosividade (Figuras 4 e 5).

A validação cruzada mostrou que o modelo ajustado para a interpolação foi eficiente $(\mathrm{NSE}=0,86)$, com alto coeficiente de determinação $\left(\mathrm{R}^{2}=0,83\right)$ e erro médio absoluto (MAE) de 9,6\%, tendo atingido a melhor acurácia diante de outras contribuições. A estimativa anterior, desenvolvida por Mello et al. (2013), obteve coeficiente de determinação $\left(\mathrm{R}^{2}\right)$ entre 0,68 e 0,75 (calculados regionalmente) e erro médio absoluto entre 7,7 e 14,1\% (calculado regionalmente). O trabalho de Silva (2004) não apresentou nenhum tipo de avaliação de sua interpolação. Logo, os mapas de erosividade obtidos no presente estudo servem como bons indicativos de locais e períodos, em que práticas adequadas de uso e manejo do solo são essenciais para evitar a perda de solo por erosão hídrica.

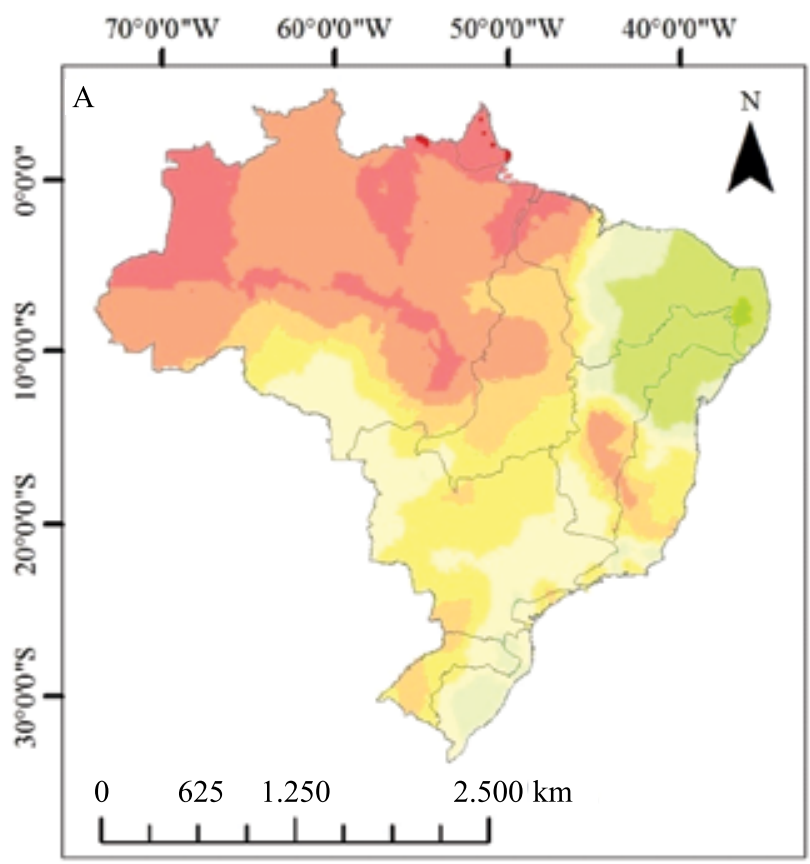

Fator $\mathrm{R}\left(\mathrm{MJ} \mathrm{mm} \mathrm{ha} \mathrm{h}^{-1} \mathrm{~h}^{-1}\right.$ por ano)

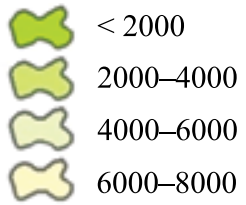

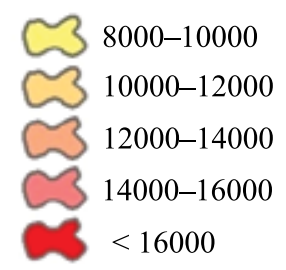

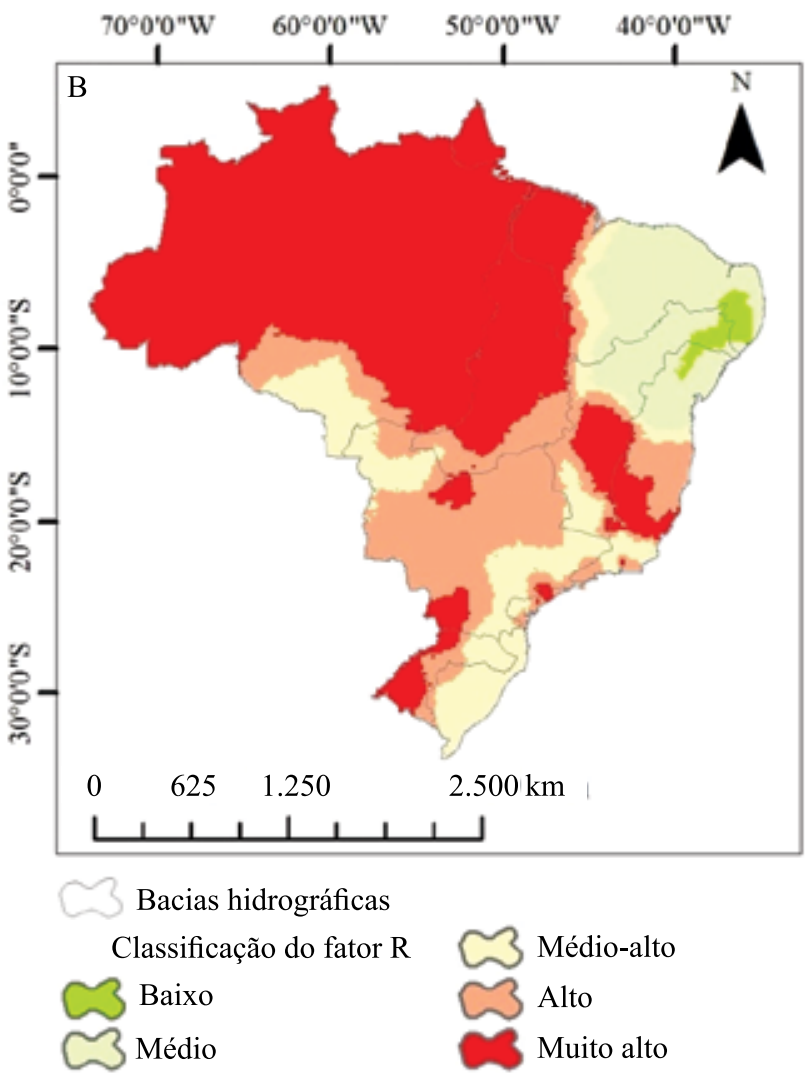

Figura 4. A, mapa do índice de erosividade das chuvas (fator R) ( $\mathrm{MJ}$ mm ha ${ }^{-1} \mathrm{~h}^{-1}$ por ano) do Brasil; e B, sua classificação de acordo com Foster et al. (1981). 

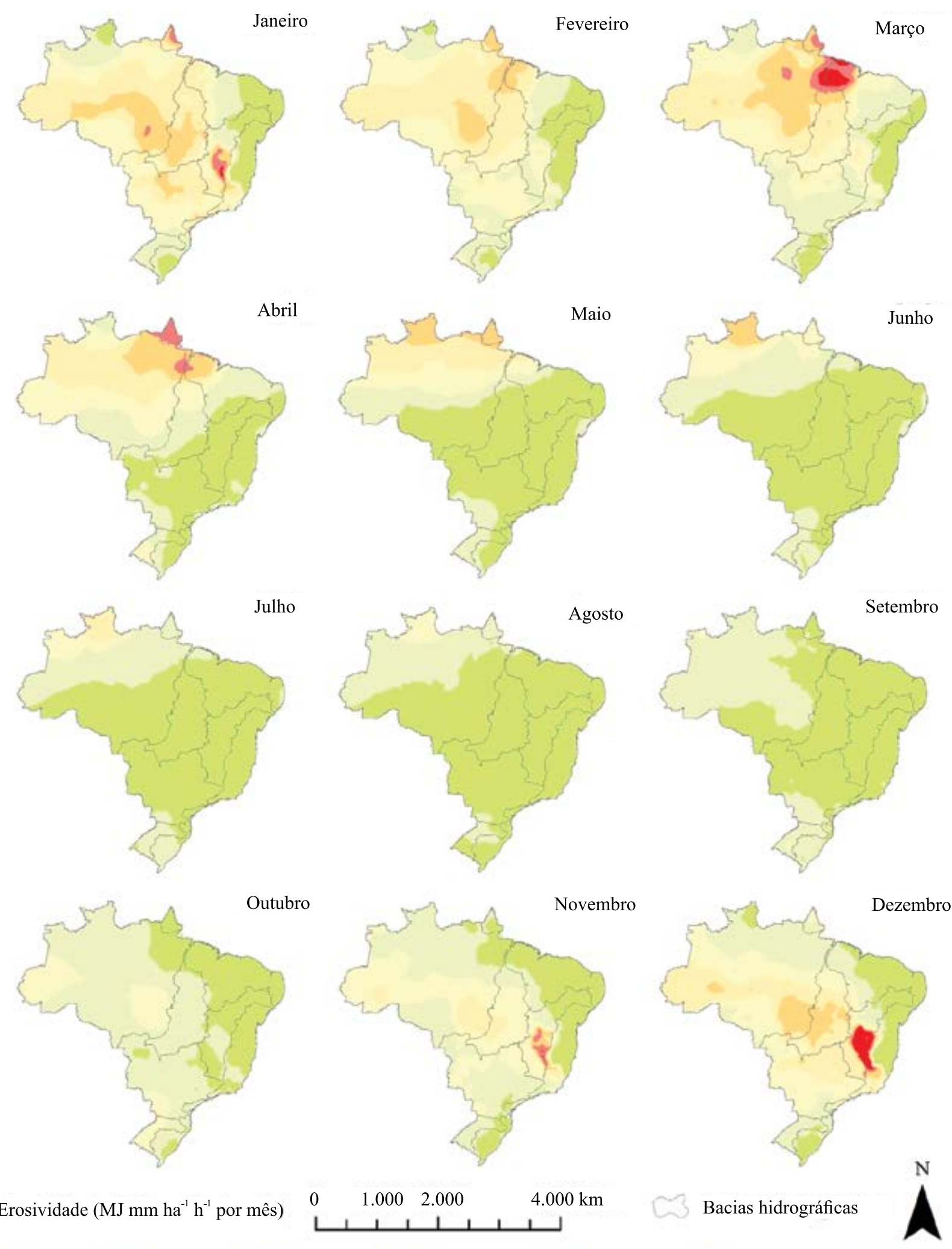

Erosividade (MJ mm ha ${ }^{-1} \mathrm{~h}^{-1}$ por mês)

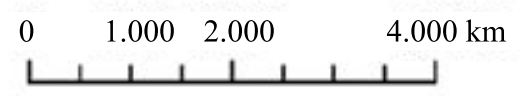

$\square 0-500$ $500-1000$ $1000-1500$ $1500-2000$ $2000-2500$

Figura 5. Variação da erosividade média mensal do Brasil ao longo do ano. 


\section{Conclusões}

1. O novo mapa de erosividade do Brasil apresenta $56 \%$ do País com fator $\mathrm{R}$ alto ou muito alto $\mathrm{e}$ com distribuição mais heterogênea das classes de erosividade.

2. A espacialização da erosividade média anual permite identificar os locais onde os índices pluviométricos são altos praticamente o ano todo e que, portanto, merecem atenção quanto às práticas conservacionistas do solo.

\section{Agradecimentos}

À Agência Nacional de Águas (ANA), pela disponibilização dos dados pluviométricos; e à Fundação de Amparo à Pesquisa do Estado de São Paulo (Fapesp, Processo 15/05134-0), pelo apoio financeiro.

\section{Referências}

ALMEIDA, C.O.S.; AMORIM, R.S.S.; ELTZ, F.L.F.; COUTO, E.G.; JORDANI, S.A. Erosividade da chuva em municípios do Mato Grosso: distribuição sazonal e correlações com dados pluviométricos. Revista Brasileira de Engenharia Agrícola e Ambiental, v.16, p.142-152, 2012. DOI: 10.1590/S141543662012000200003 .

BIASUTTI, M.; SEAGER, R. Projected changes in US rainfall erosivity. Hydrology and Earth System Sciences, v.19, p.29452961, 2015. DOI: 10.5194/hess-19-2945-2015.

BRASIL. Ministério da Agricultura, Pecuária e Abastecimento. Exportações do agronegócio crescem quase $37 \%$ em fevereiro. 2016. Disponível em: <http://www.agricultura.gov. br/comunicacao/noticias/2016/03/exportacoes-do-agronegociocrescem-quase-37porcento-em-fevereiro $>$. Acesso em: 18 mar. 2016.

CARVALHO, D.F. de; DURIGON, V.L.; ANTUNES, M.A.H.; ALMEIDA, W.S. de; OLIVEIRA, P.T.S. de. Predicting soil erosion using Rusle and NDVI time series from TM Landsat 5. Pesquisa Agropecuária Brasileira, v.49, p.215-224, 2014. DOI: 10.1590/S0100-204X2014000300008.

DIODATO, N.; KNIGHT, J.; BELLOCCHI, G. Reduced complexity model for assessing patterns of rainfall erosivity in Africa. Global and Planetary Change, v.100, p.183-193, 2013. DOI: 10.1016/j.gloplacha.2012.10.016.

ELTZ, F.L.F.; CASSOL, E.A.; PASCOTINI, P.B.; AMORIM, R.S.S. Potencial erosivo e características das chuvas de São Gabriel, RS, de 1963 a 1993. Revista Brasileira de Engenharia Agrícola e Ambiental, v.17, p.647-654, 2013. DOI: 10.1590/ S1415-43662013000600011.
FOSTER, G.R.; MCCOOL， D.K.; RENARD， K.G.; MOLDENHAUER, W.C. Conversion of the universal soil loss equation to SI metric units. Journal of Soil and Water Conservation, v.36, p.355-359, 1981.

HIDROWEB: sistema de informações hidrológicas. 2015. Disponível em: $<$ http://hidroweb.ana.gov.br/default.asp $>$. Acesso em: 25 jul. 2016.

HOOMEHR, S.; SCHWARTZ, J.S.; YODER, D.C. Potential changes in rainfall erosivity under GCM climate change scenarios for the southern Appalachian region, USA. Catena, v.136, p.141151, 2016. DOI: 10.1016/j.catena.2015.01.012.

KIRBY, M.J.; IMESON, A.C.; BERGKAMP, G.; CAMMERAAT, L.H. Scaling up processes and models from the field plot to the watershed and regional areas. Journal of Soil and Water Conservation, v.51, p.391-396, 1996.

LEE, J.-H.; HEO, J.-H. Evaluation of estimation methods for rainfall erosivity based on annual precipitation in Korea. Journal of Hidrology, v.409, p.30-48, 2011. DOI: 10.1016/j. jhydrol.2011.07.031.

MAZURANA, J.; CASSOL, E.A.; SANTOS, L.C. dos; ELTZ, F.L.F.; BUENO, A.C. Erosividade, padrões hidrológicos e período de retorno das chuvas erosivas de Santa Rosa (RS). Revista Brasileira de Engenharia Agrícola e Ambiental, v.13, p.975983, 2009. DOI: 10.1590/S1415-43662009000700021.

MELLO, C.R. de; SÁ, M.A.C. de; CURI, N.; MELLO, J.M. de; VIOLA, M.R.; SILVA, A.M. da. Erosividade mensal e anual da chuva no Estado de Minas Gerais. Pesquisa Agropecuária Brasileira, v.42, p.537-545, 2007. DOI: 10.1590/S0100204X2007000400012.

MELLO, C.R.; VIOLA, M.R.; BESKOW, S.; NORTON, L.D. Multivariate models for annual rainfall erosivity in Brazil. Geoderma, v.202-203, p.88-102, 2013. DOI: 10.1016/j. geoderma.2013.03.009.

MELO, D. de C.D.; XAVIER, A.C.; BIANCHI, T.; OLIVEIRA, P.T.S.; SCANLON, B.R.; LUCAS, M.C.; WENDLAND, E. Performance evaluation of rainfall estimates by TRMM Multisatellite Precipitation Analysis 3B42V6 and V7 over Brazil. Journal of Geophysical Research, v.120, p.9426-9436, 2015. DOI: $10.1002 / 2015 j \mathrm{jd} 023797$.

MONTANARELLA, L. Trends in land degradation in Europe. In: SIVAKUMAR, M.V.K.; NDIANG'UI, N. Climate and land degradation. New York: Springer, 2007. p.83-104. DOI: 10.1007/978-3-540-72438-4_5.

MONTEBELlER, C.A.; CEDDIA, M.B.; CARVALHO, D.F. de; VIEIRA, S.R.; FRANCO, E.M. Variabilidade espacial do potencial erosivo das chuvas no Estado do Rio de Janeiro. Engenharia Agrícola, v.27, p.426-435, 2007. DOI: 10.1590/ S0100-69162007000300011.

OLIVEIRA, P.T.S.; NEARING, M.A.; WENDLAND, E. Orders of magnitude increase in soil erosion associated with land use change from native to cultivated vegetation in a Brazilian savannah environment. Earth Surface Processes and Landforms, v.40, p.1524-1532, 2015. DOI: 10.1002/esp.3738. 
OLIVEIRA, P.T.S.; RODRIGUES, D.B.B.; SOBRINHO, T.A.; CARVALHO, D.F. de; PANACHUKI, E. Spatial variability of the rainfall erosive potential in the state of Mato Grosso do Sul, Brazil. Engenharia Agrícola, v.32, p.69-70, 2012. DOI: 10.1590/ S0100-69162012000100008.

OLIVEIRA, P.T.S.; WENDLAND, E.; NEARING, M. A. Rainfall erosivity in Brazil: a review. Catena, v.100, p.139-147, 2013. DOI: 10.1016/j.catena.2012.08.006.

PANAGOS, P.; BALLABIO, C.; BORRELLI, P.; MEUSBURGER, K.; KLIK, A.; ROUSSEVA, S.; TADIĆ, M.P.; MICHAELIDES, S.; HRABALÍKOVÁ, M.; OLSEN, P.; AALTO, J.; LAKATOS, M.; RYMSZEWICZ, A.; DUMITRESCU, A.; BEGUERÍA, S.; ALEWELL, C. Rainfall erosivity in Europe. Science of The Total Environment, v.511, p.801-814, 2015. DOI: 10.1016/j. scitotenv.2015.01.008.

PIMENTEL, D. Soil erosion: a food and environmental threat. Environment, Development and Sustainability, v.8, p.119-137, 2006. DOI: $10.1007 / \mathrm{s} 10668-005-1262-8$.

PIMENTEL, D.; HARVEY, C.; RESOSUDARMO, P.; SINCLAIR, K.; KURZ, D.; MCNAIR, M.; CRIST, S.; SPHRITZ, L.; FITTON, L.; SAFFOURI, R.; BLAIR, R. Environmental and economic costs of soil erosion and conservation benefits. Science, v.267, p.1117-1123, 1995. DOI: 10.1126/science.267.5201.1117.

RENARD, K.G.; FOSTER G.R.; WEESIES, G.A.; MCCOOL, D.K.; YODER, D.C. Predicting soil erosion by water: a guide to conservation planning with the revised universal soil loss equation (RUSLE). Washington: United States Department of Agriculture, 1997. 384p. (USDA. Agriculture handbook, 703).
SCHMIDT, S.; ALEWELL, C.; PANAGOS, P.; MEUSBURGER, $\mathrm{K}$. Regionalization of monthly rainfall erosivity patterns in Switzerland. Hydrology and Earth System Sciences, v.20, p.4359-4373, 2016. DOI: 10.5194/hess-20-4359-2016.

SHAMSHAD, A.; AZHARI, M.N.; ISA, M.H.; WAN HUSSIN, W.M.A.; PARIDA, B.P. Development of an appropriate procedure for estimation of RUSLE $\mathrm{EI}_{30}$ index and preparation of erosivity maps for Pulau Penang in Peninsular Malaysia. Catena, v.72, p.423-432, 2008. DOI: 10.1016/j.catena.2007.08.002.

SILVA, A.M. da. Rainfall erosivity map for Brazil. Catena, v.57, p.251-259, 2004. DOI: 10.1016/j.catena.2003.11.006.

SILVA, R.B.; IORI, P.; ARMESTO, C.; BENDINI, H.N. Assessing rainfall erosivity with artificial neural networks for the Ribeira Valley, Brazil. International Journal of Agronomy, v.2010, article ID 365249, 7p., 2010. DOI: 10.1155/2010/365249.

STEWART, N. (Ed.). The value of land: Prosperous lands and positive rewards through sustainable land management. Bonn: The Economics of Land Degradation, 2015. 165p.

TELLES, T.S.; GUIMARÃES, M. de F.; DECHEN, S.C.F. The costs of soil erosion. Revista Brasileira de Ciência do Solo, v.35, p.287-298, 2011. DOI: 10.1590/S0100-06832011000200001.

URI, N.D.; LEWIS, J.A. The dynamics of soil erosion in US agriculture. The Science of the Total Environment, v.218, p.4558, 1998. DOI: 10.1016/S0048-9697(98)00198-3.

WISCHMEIER, W.H.; SMITH D.D. Predicting rainfall erosion losses: a guide to conservation planning. Washington: United States Department of Agriculture, 1978. 67p. (USDA. Agriculture handbook, 537).

Recebido em 13 de maio de 2016 e aprovado em 25 de julho de 2016 